\title{
Integral-Field Spectroscopic Surveys of Nearby Early-type Galaxies
}

\author{
Eric Emsellem ${ }^{1,2}$ \\ ${ }^{1}$ European Southern Observatory, \\ 85748 Garching bei Muenchen, Germany \\ ${ }^{2}$ Université de Lyon, Observatoire de Lyon, CRAL, CNRS \\ 69563 Saint-Genis Laval, France \\ email: eric.emsellem@eso.org
}

\begin{abstract}
I briefly review here a few past or on-going surveys of nearby ETGs via integral-field spectroscopy, focusing on their dynamical status, evolution, and stellar/gaseous content.
\end{abstract}

Keywords. instrumentation: spectrographs, surveys, galaxies: elliptical and lenticular, galaxies: kinematics and dynamics, galaxies: evolution, galaxies: formation

\section{Introduction}

Early-type galaxies (ETGs) represent about $40 \%$ of the stellar mass locked in nearby galaxies (Bernardi et al. 2010), and are usually thought to be represented by two main families, namely the lenticulars (S0s) which are bulge-dominated disk systems, and the spheroid-shaped ellipticals (E's). ETGs are missing the large amplitude spiral structure (see Philippe Amram's review for an account on spirals), are overall redder than spirals, exhibit relatively small fractions of young stars and have little or no on-going star formation. Such a classification may, however, be ill-defined because it is based on morphology alone, a potential source of confusion for studies conducted at higher redshifts.

The full story of the formation/evolution of galaxies, and more specifically of ETGs, should contain a fair number of ingredients. Mergers, interactions, harassment, stripping, have been studied for decades and are often mentioned as prime contributors in the making of ETGs. Dynamical (secular) evolution due to e.g., density waves are usually associated with more disky systems. And a few processes have gained relative importance in the last decade or so (disk instabilities in gas-rich disks, cold accretion at high redshift), are now being scrutinized (shocks, turbulence, small-scale physics). The goal is to pin down the relative importance of each of these mechanisms, via signatures observed in the presumed progenitors or actual remnants, always remembering that for a stellar galactic system, formation and assembly are often not simultaneous (e.g. De Lucia et al. 2006).

Are ETGs a physically meaningul and self-consistent family of objects? What are the main ingredients for their formation/assembly and when do these enter the recipe? Is any of this relevant to understanding the history path of other galaxy classes? Integral-field spectrography of ETGs should help clarifying the picture: the associated two-dimensional spatial coverage is consistent with the apparent shape of ETGs, and the spectroscopic information is key for breaking expected degeneracies in the recovery of e.g., their intrinsic structures (see e.g. Cappellari \& McDermid 2005; Krajnović et al. 2005).

Paradoxically, Integral-Field-Units (IFUs) surveys focusing on nearby ETGs are scarce, to say the least. In this very brief review, I will list a few of these surveys and projects, leaving it to the reader to grab the corresponding publications for further details. 


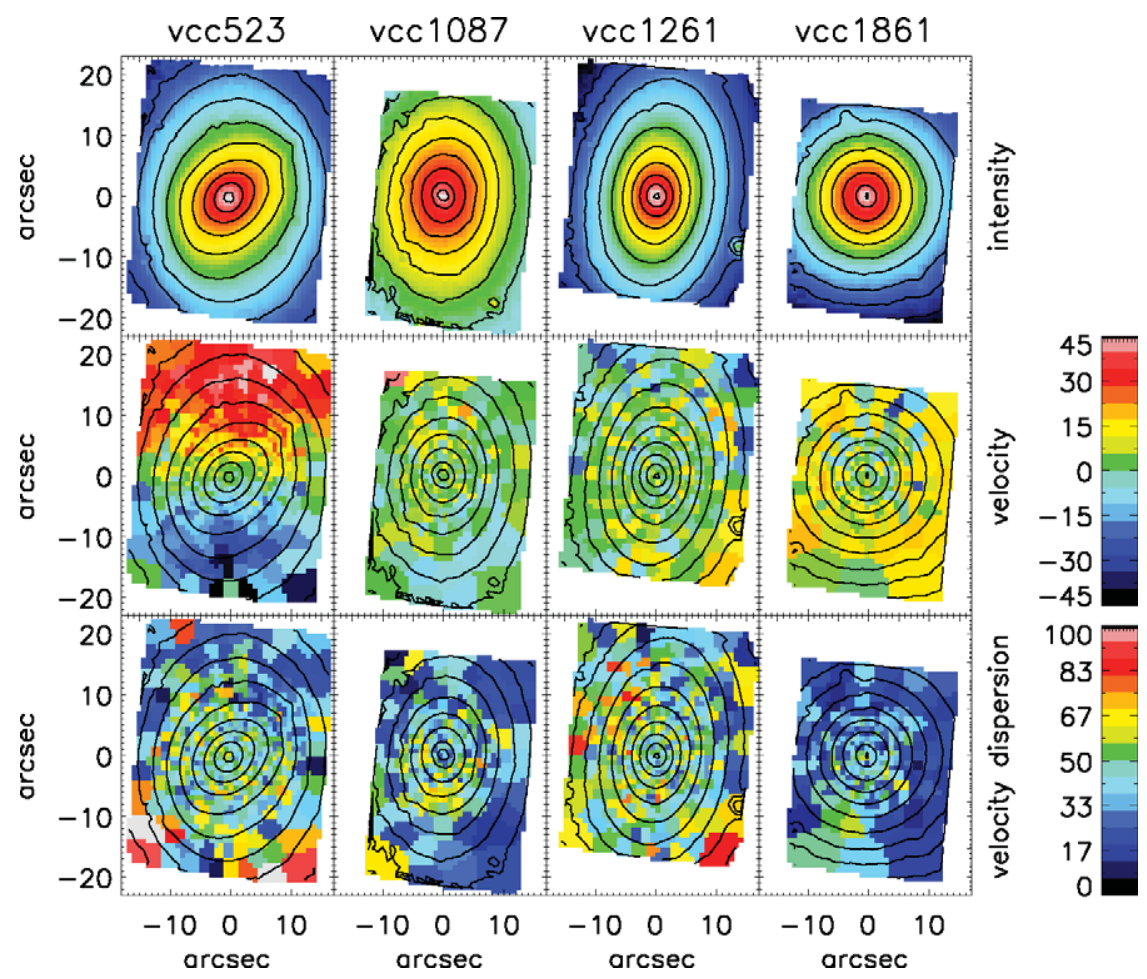

Figure 1. 4 dwarfs observed by Ryś et al. with SAURON/WHT. From top to bottom: reconstructed images, stellar velocity and velocity dispersion fields. Adapted from Ryś \& Falcón-Barroso (2010).

\section{From dwarf to massive galaxies}

At the low-mass end, data are quite rare and only a few groups targeted the challenging systems represented by dwarf galaxies. Chilingarian et al. (2007) and Chilingarian (2009) observed a few dwarfs ellipticals with e.g., MPFS/SA0-6m, but also with PMAS/CalarAlto and FLAMES/VLT (see e.g. Chilingarian et al. 2011, and references therein), revealing in some cases the presence of dynamically decoupled central components. This is also beautifully illustrated by the case of NGC 770 studied by Geha, Guhathakurta, \& van der Marel (2005) with GMOS/Gemini. Agnieszka Ryś is leading a project with Jesús Falcón-Barroso to observe a few Virgo dwarf ellipticals, extracting both the stellar kinematics and absorption line indices (Ryś \& Falcón-Barroso 2010). Preliminary results show that some of these dEs are flat but non-rotating which a priori indicates significant anisotropy (Fig. 1).

At the high-mass end, the landscape does not look more luxurious. Only a few studies have been targeting massive galaxies such as Brightest Cluster Galaxies (BCGs). Hatch, Crawford, \& Fabian (2007) observed 6 BCGs with a focus on their nebular emission with OASIS-NAOMI/WHT. They conclude that the gas configurations should be short-lived, and multiple ionisation sources be present. Edwards et al. (2009) have then obtained GMOS/Gemini and OASIS/WHT IFU spectroscopy of 9 more BCGs to again mainly map the gas distribution and kinematics and examine the various ionisation mechanisms (see also e.g. Conselice, Gallagher, \& Wyse 2001; Wilman, Edge, \& Swinbank 2006).

More recently, Sarah Brough and collaborators managed to have a first look at the stellar kinematics of 3 BCGs and their companions with VIMOS/VLT: this should allow 

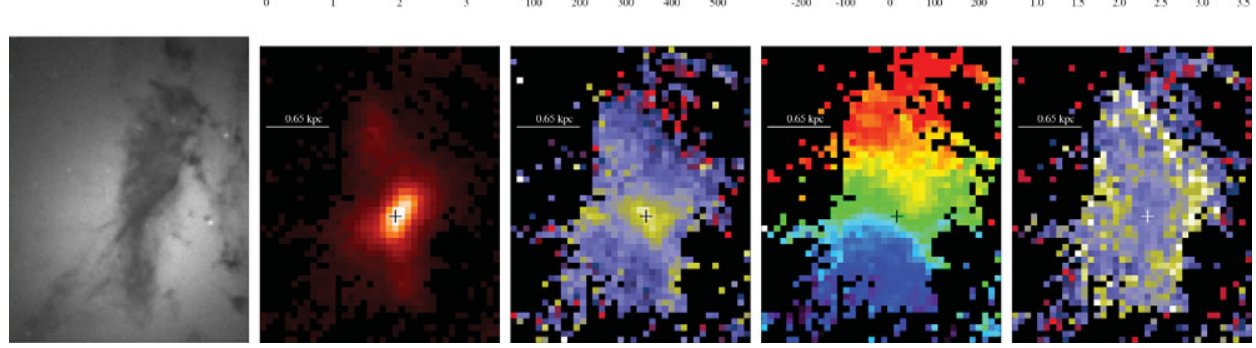

Figure 2. Images of A262. From left to right: HST ACS F435W, H $\alpha+[\mathrm{NII}]$ flux, line-width, gas velocity, and [NII]/H $\alpha$ maps. Adapted from Hatch, Crawford, \& Fabian (2007).

them to derive the apparent stellar angular momentum and correspondingly classify these galaxies as either slow or fast rotators (Emsellem et al. 2007). Rawle et al. (2008) and Rawle, Smith, \& Lucey (2010) probed the core of A3389 and of the Shapley surpercluster also with VIMOS/VLT, and examined the metallicity gradients of more than 30 galaxies, finding that there seems to be a correlation between these gradients and the measured central values. A few campaigns are still on-going, with e.g. observations of galaxies in dense environments such as Coma and A1689 with SWIFT/Palomar and FLAMES/VLT (Davies, d'Eugenio, Scott, Houghton, Dalla Bonta, et al.; Scott et al. 2011). More thorough coverage of both populations (dwarfs and galaxies in dense environments) should be conducted before we can generalise some of the promising results obtained so far.

\section{The intermediate-mass regime}

The situation is more encouraging for ETGs in the range $-22 \lesssim M_{B} \lesssim-18$ : a few campaigns have been conducted or are on-going, covering tens or hundreds of objects. For more than a decade, ETGs have been observed by e.g., S'ilchenko, Afanasiev, Chilingarian, Moiseev, and collaborators, using MPFS/SA0-6m, mostly focusing on the central regions of these galaxies and their stellar content: a summary of most of these observations is available in Silchenko (2006). An ambitious survey started last year to cover approximately 600 nearby galaxies (of all Hubble types) within $120 \mathrm{Mpc}$ with PPAK/Calar-Alto. This project (PI Sánchez) should ultimately make use of 210 dark nights over 3 years (Sánchez et al. 2010). The use of middle-size telescopes for dedicated projects like CALIFA seems an excellent strategy, specially considering the nice setup provided by the PMAS IFU. 40 galaxies have already been observed during pilot campaigns (plus 21 objects from the main sample, late- and early-type) are already being analysed. At the time of the writing of this short paper, the survey was on hold due to a failure of the telescope mechanics: the project will very hopefully resume soon.

The first survey to target a representative sample of ETGs is the SAURON project (PIs Bacon, Davies, de Zeeuw): 48 E's/S0's (and 24 Sa's) were observed with SAURON/WHT, covering up to one effective radius for most of the systems (Bacon et al. 2001; de Zeeuw et al. 2002). The IFU data have been key to emphasise trends and features pointed out in previous photometric and long-slit studies, but have also significantly changed our view of nearby ETGs. More than 30 refereed papers have appeared since in the literature, showing that, e.g., $75 \%$ of these ETGs show clear and extended ionised gas with both external and internal origins, ionisation sources being mostly associated with the stellar component (Sarzi et al. 2010), and provided important clues regarding the distribution of metals (see e.g. Kuntschner et al. 2010, and Fig. 3). It also allowed the first kinematic classification of ETGs, demonstrating the existence of two main families : fast rotators 

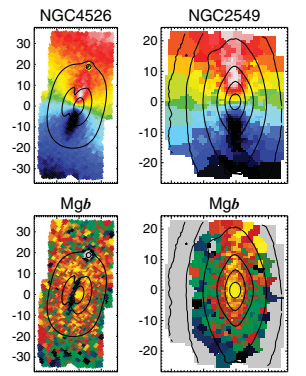

$[\mathrm{Z} / \mathrm{H}]$

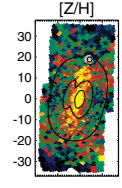

$[\alpha / \mathrm{Fe}]$

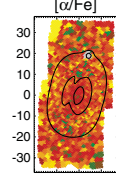

Age [Gyr]
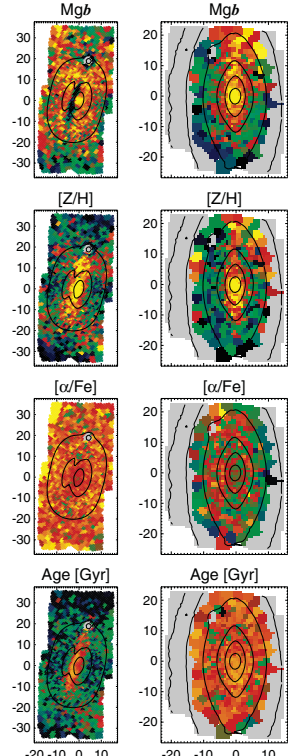

[Z/H]

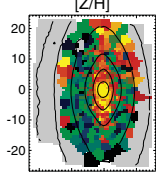

$[\alpha / \mathrm{Fe}]$

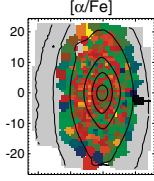

Age [Gyr]

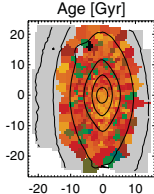

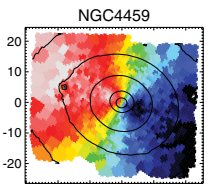

$\mathrm{Mgb}$

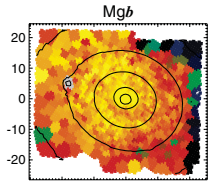

$[\mathrm{Z} / \mathrm{H}]$

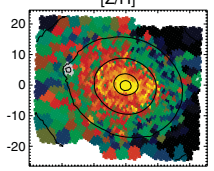

$[\alpha / \mathrm{Fe}]$

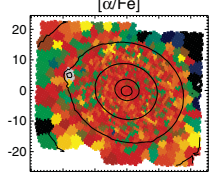

Age [Gyr]

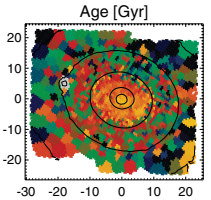

NGC4564

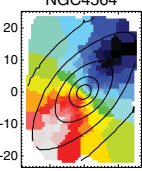

$\mathrm{Mg} b$

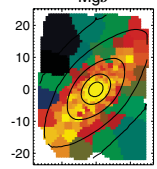

[Z/H]

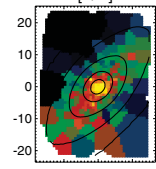

$[\alpha / \mathrm{Fe}]$

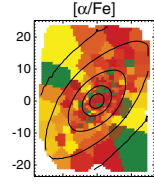

Age [Gyr]

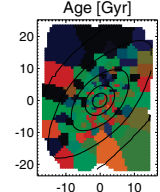

NGC4473

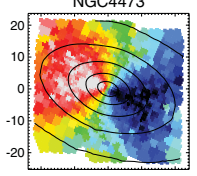

$\mathrm{Mg} b$

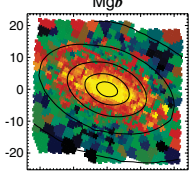

$[\mathrm{Z} / \mathrm{H}]$

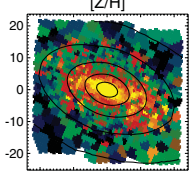

$[\alpha / \mathrm{Fe}]$

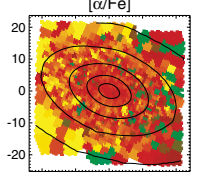

Age [Gyr]

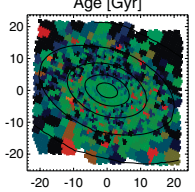

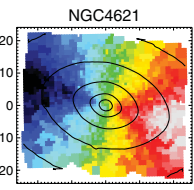

$\mathrm{Mg} b$

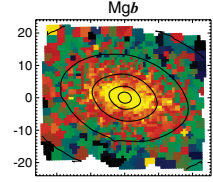

[Z/H]

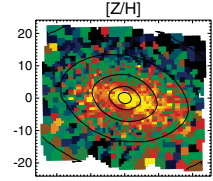

$[\alpha / \mathrm{Fe}]$

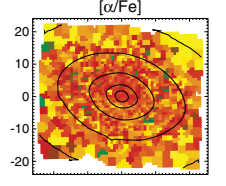

Age [Gyr]

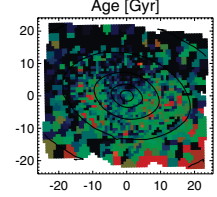

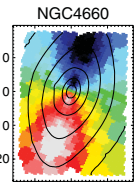

$\mathrm{Mg} b$

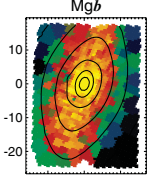

[Z/H]

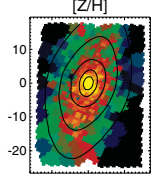

$[\alpha / \mathrm{Fe}]$

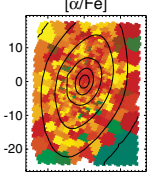

Age [Gyr]

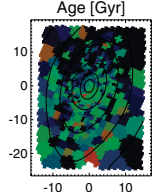

Figure 3. Prominent examples of fast rotators with a distinct, central disk-like component. ¿From top to bottom: stellar velocity field, $\mathrm{Mg} b$ line-strength, metallicity, abundance ratio $[\alpha / \mathrm{Fe}]$ and SSP-equivalent age maps. Units are given in arcsec. Extracted from Kuntschner et al. (2010).

being axisymmetric (with or without bars), generally anisotropic and disky, while slow rotators are more spherical or mildly triaxial systems, often with large photometric versus kinematic misalignments, and/or kinematically decoupled cores (Emsellem et al. 2007; Cappellari et al. 2007).

\section{The next steps}

An important milestone has been met with the ATLAS ${ }^{3 \mathrm{D}}$ survey (PIs Cappellari, Emsellem, Krajnović, McDermid) covering the first volume-limited (< $42 \mathrm{Mpc}$ ) sample of nearby ETGs, with 260 targeted galaxies above a stellar mass $M_{\star} \gtrsim 5 \times 10^{9} M_{\odot}$ (Cappellari et al. 2011). This project combines multi-wavelength information (e.g., photometry, CO single-dish/interferometric data, HI) including SAURON integral-field data, with state-of-the-art modelling and numerical simulations (e.g., binary mergers, Jean's modelling, see Bois et al. 2010; Cappellari 2008). It will provide an unprecedented view at the building block of ETGs (stars, molecular, ionised and neutral gas) including dark matter, the presence of which should be constrained via detailed stellar/gas kinematics at large radii using e.g. SAURON/WHT or VIRUS-P/H.J. Smith Telescope. An example of another way to probe large radii of ETGs is illustrated in Fig. 4 (Weijmans et al. 2009).

By the time the present manuscript is published, a number of ATLAS ${ }^{3 \mathrm{D}}$ papers should have appeared in the refereed literature, leading to a significantly updated understanding of the dynamics and chemical status of nearby ETGs. Existing IFUs and coming facilities such as KMOS and MUSE on the VLT, or the ambitious VIRUS spectrograph, 


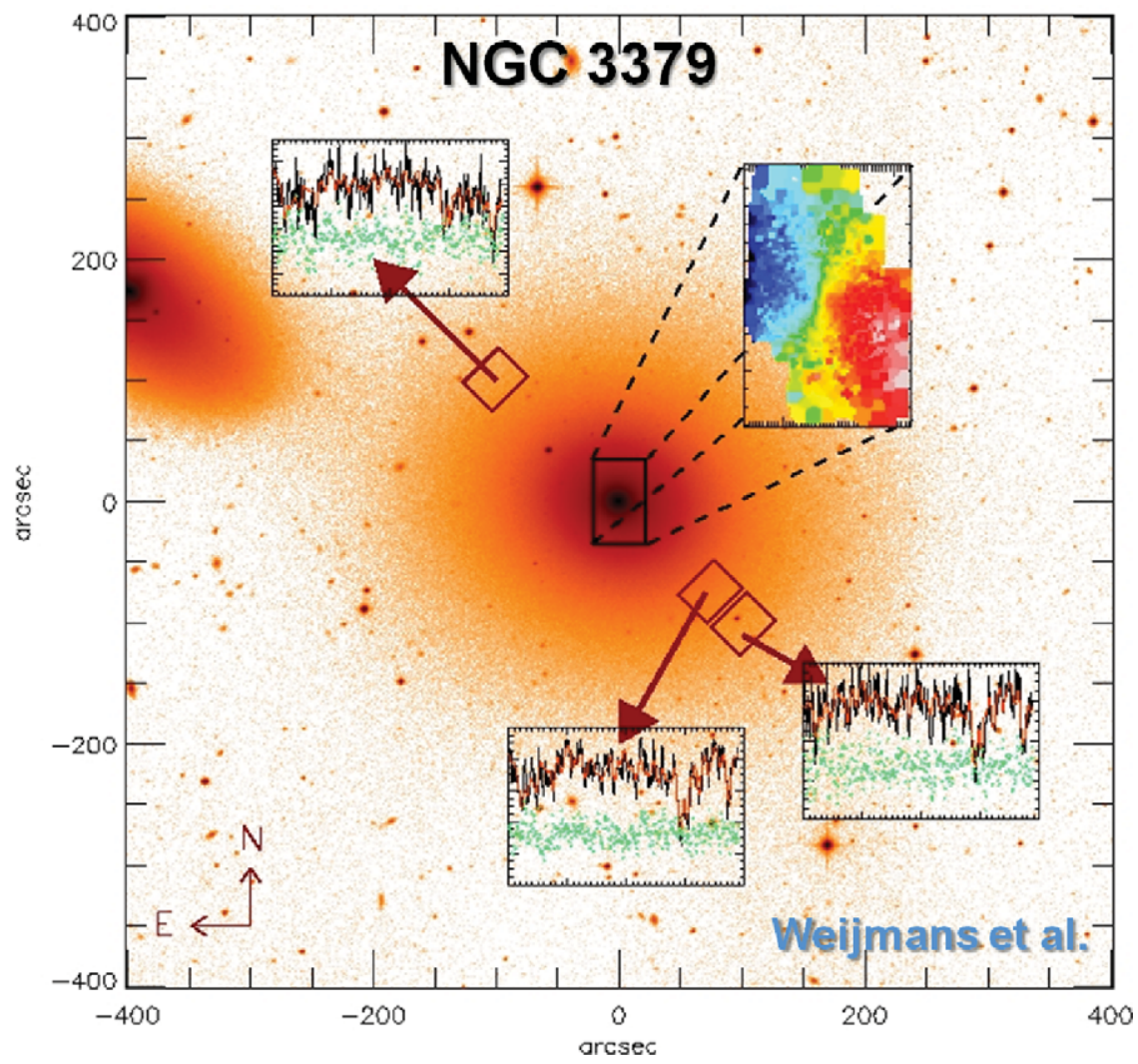

Figure 4. Using an IFU as a photon collector: the specific example of NGC 3379. The background image has been collected at the MDM Observatory and the inserted boxes show the SAURON fields of view used to probe the galaxy at several effective radii with the reduced spectra shown in black, a fit using templates in red, and the residuals in green. The central panel shows the SAURON stellar velocity field (Emsellem et al. 2004). Adapted from Weijmans et al. (2009).

are paramount to probing the wild zoo of structures witnessed in these galaxies, and given that these are accompanied by corresponding modelling efforts, large and complete samples like that of ATLAS ${ }^{3 \mathrm{D}}$ represent firm nodes with which to link the higher redshift systems.

Acknowledgements. I would like to warmly thank the SOC and its chair Claude Carignan for the invitation to give this review talk at the first West-African IAU conference. I thank my collaborators for their support, and more specifically Agnieszka Ryś, Jesús Falcón-Barroso, Nina Hatch, Sarah Brough, Sebastian Sánchez, Michele Cappellari, Richard McDermid, Davor Krajnović and Anne-Marie Weijmans, for fruitful discussions and for letting me use their nice figures, sometimes prior to publication. Greetings also to Thierry Contini for digital memories of an unforgettable musical (and knocking-over) afternoon.

\section{References}

Bacon, R., Copin, Y., Monnet, G., et al., 2001, MNRAS, 326, 23

Bernardi, M., Shankar, F., Hyde, J. B., Mei, S., Marulli, F., \& Sheth, R. K., 2010, MNRAS, 404, 2087 
Bois, M., Bournaud, F., Emsellem, E., et al., 2010, MNRAS, 406, 2405

Cappellari, M. \& McDermid, R. M., 2005, CQGra, 22, 347

Cappellari, M., Emsellem, E., Bacon, R., et al., 2007, MNRAS, 379, 418

Cappellari, M., 2008, MNRAS, 390, 71

Cappellari, M., Emsellem, E., Krajnović, D., McDermid, R., et al., 2011, MNRAS, in press (arXiv:1012.1551)

Chilingarian, I. V., Sil'Chenko, O. K., Afanasiev, V. L., \& Prugniel, P., 2007, AstL, 33, 292

Chilingarian, I. V., 2009, MNRAS, 394, 1229

Chilingarian, I. V., Mieske, S., Hilker, M., \& Infante, L., 2011, MNRAS, 66

Conselice, C. J., Gallagher, J. S., III, \& Wyse, R. F. G., 2001, AJ, 122, 2281

De Lucia, G., Springel, V., White, S. D. M., Croton, D., \& Kauffmann, G., 2006, MNRAS, 366, 499

de Zeeuw, P. T., Bureau, M., Emsellem, E., et al., 2002, MNRAS, 329, 513

Edwards, L. O. V., Robert, C., Mollá M., \& McGee S. L., 2009, MNRAS, 396, 1953

Emsellem, E., et al., 2004, MNRAS, 352, 721

Emsellem E., Cappellari, M., Krajnović, D., et al., 2007, MNRAS, 379, 401

Geha, M., Guhathakurta, P., \& van der Marel, R. P., 2005, AJ, 129, 2617

Hatch, N. A., Crawford, C. S., \& Fabian, A. C., 2007, MNRAS, 380, 33

Krajnović, D., Cappellari, M., Emsellem, E., et al., 2005, MNRAS, 357, 1113

Kuntschner, H., Emsellem, E., Bacon, R., et al., 2010, MNRAS, 408, 97

Rawle, T. D., Smith, R. J., \& Lucey, J. R., 2010, MNRAS, 401, 852

Rawle, T. D., Smith, R. J., Lucey, J. R., \& Swinbank, A. M., 2008, MNRAS, 389, 1891

Ryś A. \& Falcón-Barroso J., 2010, arXiv, arXiv:1012.0185

Sánchez, S. F., et al., 2010, arXiv, arXiv:1012.3002

Sarzi, M., Shields, J. C., Schawinski, K., et al., 2010, MNRAS, 402, 2187

Scott, N., Houghton, R., Davies, R. L., et al., 2011, AAS, 43, 14808

Silćhenko, O. K., 2006, ApJ, 641, 229

Weijmans, A.-M., et al., 2009, MNRAS, 398, 561

Wilman, R. J., Edge, A. C., \& Swinbank, A. M., 2006, MNRAS, 371, 93 